\title{
Exploring the inner space of outer space: multi-length scale, multimodal characterization of Muonionalusta IVA iron meteorite
}

Tirzah Abbott ${ }^{1}$, Stephanie Ribet ${ }^{2}$, Nathaniel Kabat ${ }^{3}$, Paul Smeets ${ }^{4}$, Roberto dos Reis ${ }^{5}$ and Vinayak Dravid $^{5}$

${ }^{1}$ NUANCE Center, Northwestern University, Evanston, Illinois, United States, ${ }^{2}$ Evanston, Illinois, United States, ${ }^{3}$ NUANCE Center, Northwestern University, Illinois, United States, ${ }^{4}$ NUANCE Center, Northwestern University, United States, ${ }^{5}$ Department of Materials Science and Engineering, Northwestern University, United States

Dual-Beam Scanning Electron Microscope/Focused Ion Beam instrumentation (FIB-SEM) provides an invaluable "one-stop-shop" for analyzing and preparing a large variety of materials for multi-scale microanalysis. The addition of electron backscatter diffraction (EBSD) detectors in a FIB-SEM have allowed for in situ sample preparation and analysis at multiple length scales in one single instrument. In this study, we have applied a suite of electron microscopy scattering techniques to understand the orientation, grain size, and grain boundary strain at different scales in the hierarchal structures within the Muonionalusta IVA octahedrite iron meteorite. The JEOL-4700F Multi Beam FIB-SEM equipped with the Oxford Instruments Symmetry CMOS EBSD detector was used to perform micron scale EBSD, transmission Kikuchi diffraction (TKD), and 3D EBSD of the Muonionalusta IVA iron meteorite. The prepared TEM lamella in the FIB-SEM can then be characterized with a number of STEM techniques to elucidate nano-scale crystallographic information.

Microstructures in iron meteorites that have fallen on the surface of the Earth provide a window into the history of the Universe. Iron meteorites (IVB/IVA) in particular, characterized by interlocking plates of kamacite $(\alpha-B C C$, ferrite) and taenite $(\gamma-F C C$, austenite) crystals in a Widmanstatten pattern, are especially important due to their utility in thermal history of asteroidal bodies. ${ }^{1}$ The matrix surrounding these plates is composed of plessite, a mixture of taenite and kamacite ${ }^{1,2}$. Electron backscatter diffraction in a scanning electron microscope has been used to understand micron-scale grain size and orientation of the Widmanstatten patterned phases to determine the cooling history of the meteorite after separating from the parent body ${ }^{3}$. However, the story hidden in the plessite matrix requires higher resolution crystallographic characterization, making these iron meteorites a perfect example of the need for a comprehensive, multi-length scale analytical approach.

Backscattered electron SEM imaging (showing the compositional contrast between grains) and EBSD (displaying phase and orientation) (Figure 1) shows the hierarchal structure of kamacite/taenite plates and the complex plessite matrix with taenite nodules in 2D (Figure 1a, 1b). The plessite was also serial sectioned with the FIB-SEM, which is integrated into the Oxford AZtec interface for 3D EBSD analysis. Traditional EBSD is unable to resolve a resolution below approx. $20 \mathrm{~nm}$ due to the large beam-specimen interactions on the highly tilted (70 degree) sample surface ${ }^{4}$. Thin lamella (approx. $80 \mathrm{~nm}$ ) of the plessite containing interstitial areas were marked and extracted from a region of interest (ROI) using the FIBSEM, mounted onto Cu FIB grids, and mapped using transmission kikuchi diffraction (Figure 1c) in order to observe any smaller grains. The TKD is able to display multiple grains of different orientation ranging from 20-50 nm, but is unable to show strain deformation at the grain boundaries (Figure 1d).

Scanning transmission electron microscopy (STEM) provides a valuable correlative tool for characterizing these meteorites due to its nano- to atomic scale resolution and multimodal signal acquisition. In particular, four-dimensional STEM (4D-STEM), is a method where at each position in real space, an electron diffraction pattern is collected, containing the rich phase-space chemical and physical information about 
the sample. Through a variety of reconstruction techniques, this data can be used to map strain and grain structure in addition to other physiochemical properties. In order to unravel the complex structure of the regions of the Muonionalusta IVA with nanoscale features, our ongoing studies are using a suite of STEM techniques to characterize its architecture.

This presentation will provide insights into the Muonionalusta IVA architecture using multimodal, multiple length scale characterization. More broadly, we demonstrate how a FIB-SEM can be instrumental to the study of such a sample with a variety of cutting-edge electron microscopy techniques. ${ }^{5}$
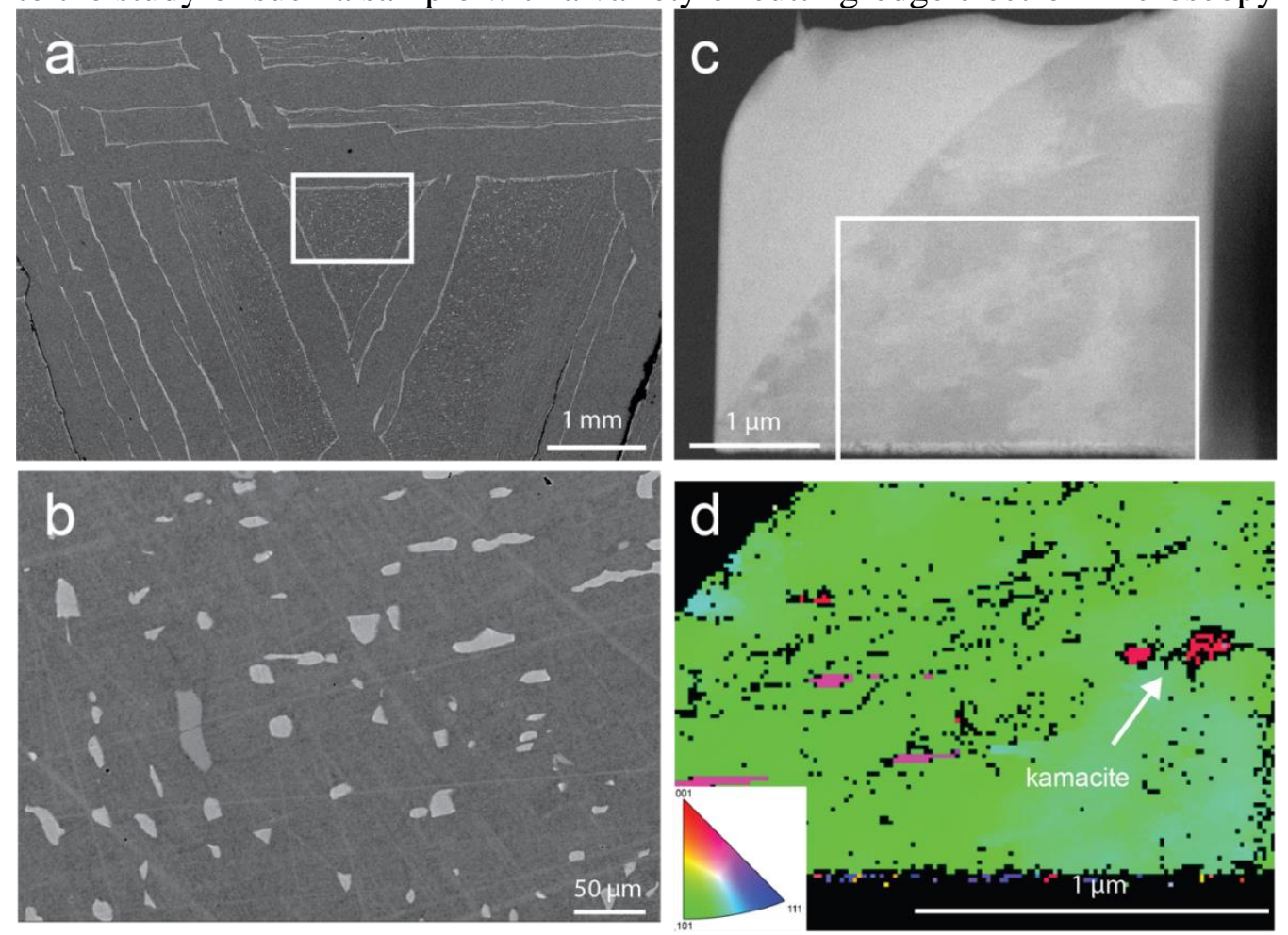

Figure 1. Backscattered electron image of interlocking plates of kamacite ( $\alpha$-bcc, ferrite) and taenite $(\gamma$ fcc, austenite) crystals in a Widmanstatten pattern and (b) plessite, a mixture of taenite and kamacite, interstitial matrix. An approx. $80 \mathrm{~nm}$ thick lamella was prepared using conventional lift-out (c) to perform using TKD. The inverse pole figures (d) show the different crystallographic orientation of grains (according to color scheme in map) showing most kamacite oriented in the same direction but including smaller, nano-meter sized grains. 4D-STEM using a direct electron detector (K3 IS) to collect diffraction patterns at every pixel position will be utilized to characterize smaller scale grain boundary strain and misorientation.

\section{References}

1. Rasmussen, K. L.; Malvin, D. J.; Wasson, J. T., Trace Element Partitioning between Taenite and Kamacite; Relationship to the Cooling Rates of Iron Meteorites. Meteoritics 1988, 23 (2), 107-112.

2. Goldstein, J. I.; Michael, J. R., The formation of plessite in meteoritic metal. Meteoritics \& Planetary Science 2006, 41 (4), 553-570.

3. Goldstein, J. I.; Yang, J.; Kotula, P. G.; Michael, J. R.; Scott, E. R. D., Thermal histories of IVA iron meteorites from transmission electron microscopy of the cloudy zone microstructure. Meteoritics \& Planetary Science 2009, 44 (3), 343-358.

4. Sneddon, G. C.; Trimby, P. W.; Cairney, J. M., Transmission Kikuchi diffraction in a scanning electron microscope: A review. Materials Science and Engineering: R: Reports 2016, 110, 1-12. 
5. This work made use of the EPIC facility of Northwestern University's NUANCE Center, which has received support from the SHyNE Resource (NSF ECCS-2025633), the IIN, and Northwestern's MRSEC program (NSF DMR-1720139). This work will be incorporated in efforts to create a collaborative and comprehensive instrumentation suite and facilities for extra-terrestrial materials as described in "Infrastructure needs for Extra-Terrestrial Materials" published by the National Academies Science (https://www.nap.edu/read/25312/chapter/1) 